Pacific Journal of Mathematic 


\title{
SHIFTS ON INDEFINITE INNER PRODUCT SPACES
}

\author{
BRIAN W. MCENNIS
}

We use the concept of a wandering subspace to study isometries on spaces with an inner product that is not assumed to be positive definite. The theory in many respects parallels the Hilbert space theory, but there are significant differences that are emphasized here. Examples are given which illustrate the complications that can arise when the inner product is indefinite.

The first few sections of this paper are devoted to the study of indefinite inner product spaces with admissible topologies, and the continuous operators on these spaces. The rest of the paper concentrates on isometric operators, their wandering subspaces, and the Fourier representations of shifts.

1. Introduction. In the paper [5], Halmos studies shifts on Hilbert spaces by using wandering subspaces. We apply this technique here, where we consider isometries on indefinite inner product spaces. These operators have been studied in the past, principally by Iohvidov ([6] and [7]), on spaces where the indefinite inner product is derived from a Hilbert space inner product (a $J$ - or $G$-inner product). The results obtained for isometries here, however, apply in the more general situation of an inner product space with an admissible topology.

The theory of shifts on Hilbert space was used by Sz.-Nagy and Foias [11] in the study of the geometry of spaces of minimal unitary dilations of contractions. A noncontraction possesses a minimal unitary dilation on a space with an indefinite inner product [2], and it was the study of the geometry of these dilation spaces (originating in the papers of Davis [2] and Davis and Foias [3]) that motivated the present work.

Most of the results appearing here formed part of the author's $\mathrm{Ph}$. D. thesis [10]; other work was partially supported by a grant from the National Science Foundation.

2. Inner product spaces. An inner product space is a complex vector space $\mathscr{H}$ with an inner product $[\cdot, \cdot]$ satisfying

$$
\left[\alpha_{1} h_{\llcorner}+\alpha_{2} h_{2}, k\right]=\alpha_{1}\left[h_{1}, k\right]+\alpha_{2}\left[h_{2}, k\right]
$$

and

$$
[k, h]=\overline{[h, k]}
$$


for all $\alpha_{1}, \alpha_{2} \in C$ and for all $h_{1}, h_{2}, h, k \in \mathscr{X}$. The inner product is not assumed to be positive, that is $[h, h]$ may be negative for some $h \in \mathscr{K}$. For the theory of inner product spaces, refer to [1] and [8].

If $[h, k]=0$, then we write $h \perp k$. If $\mathscr{A}$ and $\mathscr{B}$ are two subsets of $\mathscr{H}$, then we write $h \perp \mathscr{B}$ if $h \perp k$ for all $k \in \mathscr{B}$, and $\mathscr{A} \perp \mathscr{B}$ if $h \perp \mathscr{B}$ for all $h \in \mathscr{A}$.

If $\mathscr{L}_{1}$ and $\mathscr{L}_{2}$ are two subspaces, with $\mathscr{L}_{1} \perp \mathscr{L}_{2}$ and $\mathscr{L}_{1} \cap \mathscr{L}_{2}=$ $\{0\}$, then we write $\mathscr{L}_{1} \oplus \mathscr{L}_{2}$ for the direct sum of $\mathscr{L}_{1}$ and $\mathscr{L}_{2}$.

For any subset $\mathscr{A} \subseteq \mathscr{H}$ we define

$$
\mathscr{H}^{\perp}=\{h \in \mathscr{H}: h \perp \mathscr{A}\} .
$$

Note (cf. [1, Sec. I.3]) that $\mathscr{A}^{\perp}$ is a subspace, and if $\mathscr{A} \cong \mathscr{B}$ then $\mathscr{B} \mathscr{B}^{\perp} \subseteq \mathscr{A}^{\perp}$. Also, if $\mathscr{L}_{1}$ and $\mathscr{L}_{2}$ are subspaces of $\mathscr{\mathscr { C }}$, then we have

$$
\left(\mathscr{L}_{1}+\mathscr{L}_{2}\right)^{\perp}=\mathscr{L}_{1}^{\perp} \cap \mathscr{L}_{2}^{\perp} \text {. }
$$

In contrast to the situation in Hilbert space, it is possible to have $\mathscr{L} \cap \mathscr{L}^{\perp} \neq\{0\}$ for a subspace $\mathscr{L}$. The subspace

$$
\mathscr{L}^{0}=\mathscr{L} \cap \mathscr{L}^{1}
$$

is called the isotropic part of $\mathscr{L}$. A subspace $\mathscr{L}$ of $\mathscr{H}$ is said to be degenerate if $\mathscr{L}^{0} \neq\{0\}$, i.e., if $\mathscr{L} \cap \mathscr{L}^{\leftarrow} \neq\{0\}$ ([1, Sec. I.4]).

We will be assuming throughout that all inner product spaces are nondegenerate, i.e., $\mathscr{H}^{-}=\{0\}$ for every inner product space $\mathscr{C}$. Consequently, if

$$
\left[h, k_{1}\right]=\left[h, k_{2}\right]
$$

for each $h \in \mathscr{X}$, then $k_{1}=k_{2}$.

If $\mathscr{L}_{1}$ and $\mathscr{L}_{2}$ are subspaces of $\mathscr{H}$, with $\mathscr{L}_{1} \subseteq \mathscr{L}_{2}$, then $\mathscr{L}_{2} \ominus \mathscr{L}_{1}$ will be used to denote the subspace $\mathscr{L}_{2} \cap \mathscr{L}_{1}{ }^{1}$.

We will need the following lemma. The proof is straight-forward and is omitted. (See [10, Lemma I.1.1].)

LEMma 2.1. If $\mathscr{L}=\mathscr{L}_{1} \oplus \mathscr{L}_{2}$, for subspaces $\mathscr{L}_{1}, \mathscr{L}_{2}$ of an inner product space $\mathscr{C}$, then

$$
\mathscr{L} \ominus \mathscr{L}_{1}=\mathscr{L}_{1}^{0} \oplus \mathscr{L}_{2}
$$

A topology on an inner product space $\mathscr{H}$ is said to be admissible if it is locally convex, and if (i) for every $k \in \mathscr{C}$ the linear functional

$$
\phi_{k}(h)=[h, k]
$$

is continuous, and (ii) for every continuous linear functional $\phi$ on $\mathscr{C}$ there is a $k \in \mathscr{H}$ such that $\phi=\phi_{k}$ ([1, Sec. III.5]). Every admissible 
topology on a nondegenerate space is separated, i.e., Hausdorff ([1, Lemma III.5.2]).

The weak topology on $\mathscr{\mathscr { C }}$ is the locally convex topology defined by the family $\left\{p_{k}\right\}_{k \in \mathscr{B}}$ of seminorms, where

$$
p_{k}(h)=|[h, k]| \quad(h, k \in \mathscr{C})
$$

([1, Sec. III.2]). The weak topology is admissible ([1, Theorem III.5.1]).

THeOREM 2.2. If $\mathscr{L}$ is a subspace of $\mathscr{l}$, then the closure of $\mathscr{L}$, with respect to any admissible topology, is $\mathscr{L}^{11}$.

Proof. See [1, Theorem III.6.1].

COROLLARY 2.3. A subspace of $\mathscr{H}$ is closed with respect to an admissible topology if and only if it is weakly closed.

CoRollary 2.4. If $\mathscr{L}_{1} \perp \mathscr{L}_{2}$, then $\overline{\mathscr{L}}_{1} \perp \mathscr{L}_{2}$, where $\overline{\mathscr{L}}_{1}$ denotes the closure of the subspace $\mathscr{L}_{1}$ with respect to any admissible topology.

3. Operators on inner product spaces. We assume from this point on that all spaces are nondegenerate inner product spaces with admissible topologies. An operator $T: \mathscr{C}_{1} \rightarrow \mathscr{K}_{2}$ is assumed to be linear with domain equal to $\mathscr{K}_{1}$. The identity operator will be denoted by $I$.

We will be needing the concept of a generalized sequence (or net) (see, for example, [4, Definition I.7.1]), and in particular the following lemma (cf. [4, Lemma I.7.4]):

Lemma 3.1. An operator $T$ is continuous if and only if for every generalized sequence $\left\{h_{\alpha}\right\}$ converging to zero, $\left\{T h_{\alpha}\right\}$ converges to zero.

Lemma 3.2. Suppose $\mathscr{H}$ and $\mathscr{K}^{\prime}$ are two spaces with topologies defined by the families of seminorms

$$
\left\{p_{x}\right\}_{x \in X} \text { and }\left\{p_{y}\right\}_{y \in Y},
$$

respectively. If an operator $T$ from $\mathscr{H}$ to $\mathscr{H}^{\prime}$ has the property that for each $y \in Y$ there is an $x \in X$ such that

$$
p_{y}(T h)=p_{x}(h),
$$

for every $h \in \mathscr{K}$, then $T$ is continuous.

Proof. Suppose that the generalized sequence $\left\{h_{\alpha}\right\}$ in $\mathscr{H}$ converges 
to zero. Then, for every $y \in Y$, the generalized sequence of numbers

$$
\left\{p_{y}\left(T h_{\alpha}\right)\right\}=\left\{p_{x}\left(h_{\alpha}\right)\right\}
$$

converges to zero. But this is equivalent to saying that $\left\{T h_{\alpha}\right\}$ converges to zero and so, by Lemma 3.1, $T$ is continuous.

Proposition 3.3. If $T$ is a continuous operator from $\mathscr{H}$ to $\mathscr{Y}^{\prime \prime}$, then there exists a unique operator $T^{*}$ from $\mathscr{H}^{\prime}$ to $\mathscr{H}$ such that, for each $h \in \mathscr{Y}$ and $h^{\prime} \in \mathscr{H}^{\prime}$

$$
\left[T h, h^{\prime}\right]=\left[h, T^{*} h^{\prime}\right] .
$$

$T^{*}$ is weakly continuous.

Proof. For $h^{\prime} \in \mathscr{H}^{\prime},\left[T h, h^{\prime}\right]$ is a continuous function of $h$. Since the topology on $\mathscr{H}$ is assumed to be admissible, there is a vector $k \in \mathscr{H}$ such that $\left[T h, h^{\prime}\right]=[h, k] . \quad \mathscr{H}$ is nondegenerate, and so this equation uniquely determines $k$, and we can define $T^{*} h^{\prime}=k$. Clearly, $T^{*}$ is an operator from $\mathscr{C}^{\prime}$ to $\mathscr{C}$, and it follows directly from Lemma 3.2 that $T^{*}$ is continuous in the weak topologies of $\mathscr{H}$ and $\mathscr{H}^{\prime}$.

$T^{*}$ is known as the adjoint of $T$.

Proposition 3.4. Every continuous operator is weakly continuous.

Proof. If $T$ is continuous, then $T=T^{* *}$, and by Proposition 3.3 the adjoint of any operator is weakly continuous.

4. Regular subspaces, Projections.

THEOREM 4.1. If $\mathscr{L}$ is a subspace of $\mathscr{H}$, then $\mathscr{L}+\mathscr{L}^{\perp} i s$ dense in $\mathscr{C}$ if and only if $\mathscr{L}^{\perp \perp}$ is nondegenerate.

Proof. See [1, Theorem III.6.5].

Thus if $\mathscr{L}$ is a closed subspace which is nondegenerate, then

$$
\mathscr{H}=\overline{\mathscr{L} \oplus \mathscr{L}^{\perp}} \text {. }
$$

If in fact

$$
\mathscr{H}=\mathscr{L} \oplus \mathscr{L}^{1},
$$

we call $\mathscr{L}$ a regular subspace. Note that every regular subspace is nondegenerate. 
REMARK. This definition generalizes the use of the terms regular in [3], right in [9], and pravil'nym in [7], and coincides with the use of ortho-complemented in [1], at least for nondegenerate inner product spaces.

If $\mathscr{L}$ is regular, then every $h \in \mathscr{H}$ has a unique expression in the form $h=l+l^{\prime}\left(l \in \mathscr{L}, l^{\prime} \in \mathscr{L}^{\perp}\right)$. We define $P_{\mathscr{L}}$, the projection of $\mathscr{H}$ onto $\mathscr{L}$, by

$$
P_{\mathscr{L}} h=l \text {. }
$$

Clearly, $P_{\mathscr{L}}$ is linear, $P_{\mathscr{L}}^{2}=P_{\mathscr{L}}$, and for each $h, k \in \mathscr{H}$

$$
\left[P_{\mathscr{L}} h, k\right]=\left[h, P_{\mathscr{L}} k\right] \text {. }
$$

Therefore, by Lemma $3.2, P_{\mathscr{L}}$ is weakly continuous and $P_{\mathscr{L}}^{*}=P_{\mathscr{L}}$.

It is obvious that if $\mathscr{L}$ is regular, so is $\mathscr{L}^{\perp}$ and $P_{\mathscr{L}}^{\perp}=I-P_{\mathscr{L}}$.

An operator $P$ on $\mathscr{H}$ is called a projection if it is weakly continuous and if $P^{*}=P^{2}=P$.

The proofs of the following theorem and its corollaries are straightforward, and are omitted.

TheOREM 4.2 (cf. [9, p. 116]). If $P$ is a projection, then $\mathscr{L}=$ $P \mathscr{C}$ is regular and $P=P_{\mathscr{S}}$.

COROLLARY 4.3. $\mathscr{L}$ is regular if and only if there is a projection $P$ such that $\mathscr{L}=P \mathscr{H}$.

CoRollaRY 4.4. If $P_{\mathscr{L}_{1}}$ and $P_{\mathscr{L}_{2}}$ commute, for two regular subspaces $\mathscr{L}_{1}$ and $\mathscr{L}_{2}$, then $\mathscr{L}_{3}=\mathscr{L}_{1} \cap \mathscr{L}_{2}$ is regular with $P_{\mathscr{S}_{3}}=P_{\mathscr{L}_{1}} P_{\mathscr{L}_{2}}$.

COROLLARY 4.5. If $\mathscr{L}_{1}$ and $\mathscr{L}_{2}$ are regular subspaces with $\mathscr{L}_{1} \subseteq$ $\mathscr{L}_{2}$, then $\mathscr{L}_{3}=\mathscr{L}_{2} \ominus \mathscr{L}_{1}$ is regular with $P_{\mathscr{L}_{3}}=P_{\mathscr{L}_{2}}-P_{\mathscr{L}_{1}}$.

In a Hilbert space the direct sum of two orthogonal closed subspaces is always closed. In an indefinite inner product space this is true, provided we assume that one of the subspaces is regular.

THEOREM 4.6 (cf. [9, Lemma 5.1]). Suppose $\mathscr{L}_{1}$ and $\mathscr{L}_{2}$ are subspaces of $\mathscr{H}$ satisfying $\mathscr{L}_{1} \perp \mathscr{L}_{2}$. Then if $\mathscr{L}_{1}$ is regular and $\mathscr{L}_{2}$ is closed, $\mathscr{L}_{1} \oplus \mathscr{L}_{2}$ is closed. If, in addition, $\mathscr{L}_{2}$ is regular, then $\mathscr{L}_{1} \oplus \mathscr{L}_{2}$ is regular.

Proof. Since $\mathscr{L}_{2} \subseteq \mathscr{L}_{1}^{\perp}$ and $\mathscr{L}_{1}$ is regular, the sum of $\mathscr{L}_{1}$ and $\mathscr{L}_{2}$ is direct. 
Suppose $h$ is in the closure of $\mathscr{L}_{1} \oplus \mathscr{L}_{2}$. Then, by Corollary 2.3, there is a generalized sequence $\left\{h_{\alpha}\right\}$ in $\mathscr{L}_{1} \oplus \mathscr{L}_{2}$ converging weakly to $h\left(\left[4\right.\right.$, Lemma I.7.2]). Let $P$ be the projection of $\mathscr{H}$ onto $\mathscr{L}_{1}$. Clearly, $(I-P) h_{\alpha} \in \mathscr{L}_{2}$ and, since $P$ is weakly continuous and $\mathscr{L}_{2}$ is closed, $(I-P) h \in \mathscr{L}_{2}$. Hence $h=P h+(I-P) h \in \mathscr{L}_{1} \oplus \mathscr{L}_{2}$, and $\mathscr{L}_{1} \oplus \mathscr{L}_{2}$ is therefore closed.

Now suppose $\mathscr{L}_{2}$ is regular and let $Q$ be the projection of $\mathscr{C}$ onto $\mathscr{L}_{2}$. Since $\mathscr{L}_{1} \perp \mathscr{L}_{2}, Q P=P Q=0$. Hence $P+Q$ is a projection with $(P+Q) \mathscr{C}=\mathscr{L}_{1} \oplus \mathscr{L}_{2}$ and so, by Theorem 4.2, $\mathscr{L}_{1} \oplus \mathscr{L}_{2}$ is regular.

5. Isometries. An operator $V$ from $\mathscr{H}$ to $\mathscr{H}^{\prime}$ is called an isometry if it is continuous and $[V h, V k]=[h, k]$ for each $h, k \in \mathscr{K}$. The condition that a continuous operator $V$ be an isometry is equivalent to $V^{*} V=I$, and hence every isometry is injective. (Cf. [6, Proposition $1^{\circ}$ ]. Recall that $\mathscr{C}$ is assumed to be nondegenerate.)

An isometry is called unitary if it is surjective. As in Hilbert space, the unitary operators $V$ are characterized by the relations $V^{*} V=I$ and $V V^{*}=I$, i.e., by the relation $V^{*}=V^{-1}$.

Lemma 5.1 (cf. [6, Theorem 2]). If $V$ is an isometry and $\mathscr{L}$ is a closed subspace, then $V \mathscr{L}$ is also a closed subspace.

Proof. $V \mathscr{L}$ is clearly a subspace. In view of Corollary 2.3 it suffices to show $V \mathscr{L}$ is weakly closed.

Suppose $h$ is in the weak closure of $V \mathscr{L}$. Select a generalized sequence $\left\{l_{\alpha}\right\}$ of vectors in $\mathscr{L}$ so that $\left\{V l_{\alpha}\right\}$ converges weakly to $h$. Since $V^{*}$ is weakly continuous, $\left\{l_{\alpha}\right\}=\left\{V^{*} V l_{\alpha}\right\}$ converges weakly to $V^{*} h$. By Corollary 2.3, $\mathscr{L}$. is weakly closed, so $V^{*} h \in \mathscr{L}$. Also, since Proposition 3.4 implies the weak continuity of $V,\left\{V l_{\alpha}\right\}$ converges weakly to $V V^{*} h$. But $\left\{V l_{\alpha}\right\}$ was assumed to converge weakly to $h$ and the weak topology is separated, so $h=V V^{*} h \in V \mathscr{L}$. Hence $V \mathscr{L}$ is weakly closed.

TheOREM 5.2 (cf. [7, p. 176], [1, Theorem VI.3.8]). If $\mathscr{L}$ is a regular subspace of $\mathscr{H}$ and $V$ is an isometry from $\mathscr{H}$ to $\mathscr{H}^{\prime}$, then $V \mathscr{L}$ is a regular subspace of $\mathscr{L}^{\prime}$. The projection of $\mathscr{H}^{\prime}$ onto $V \mathscr{L}$ is $V P_{\mathscr{L}} V^{*}$.

Proof. Let $P=P_{\mathscr{L}}, Q=V P V^{*}$. Then $Q$ is weakly continuous, $Q=Q^{*}$, and since $V$ is an isometry, $Q^{2}=Q$. Therefore $Q$ is a projection.

We have $Q \mathscr{C}^{\prime} \subseteq V P \mathscr{H}=V \mathscr{L}$ and for any $l \in \mathscr{L} Q V l=V P V^{*} V l=$ $V P l=V l$. Hence $Q \mathscr{H}^{\prime}=V \mathscr{L}$, so by Theorem 4.2, $V \mathscr{L}$ is regular 
and $Q$ is the projection of $\mathscr{Y}^{\prime}$ onto $V \mathscr{L}$.

We also include the following result, in which the operator $V$ is not assumed to be continuous. A similar result has been obtained by Iohvidov [7, Theorem 2] for $G$-isometric operators on a Hilbert space with a $G$-metric. Here we consider arbitrary nondegenerate inner product spaces $\mathscr{C}$ and $\mathscr{H}^{\prime}$ and we discuss the weak continuity of $V$.

THEOREM 5.3. Suppose that $V: \mathscr{H} \rightarrow \mathscr{H}^{\prime}$ satisfies $[V h, V k]=$ $[h, k]$ for all $h, k \in \mathscr{H}$. Then $V$ is weakly continuous if and only if $V \mathscr{H}$ is a regular subspace of $\mathscr{H}^{\prime}$.

Proof. If $V$ is weakly continuous, then Theorem 5.2 shows that $V \mathscr{H}$ is a regular subspace of $\mathscr{H}^{\prime}$. Conversely, suppose that $V \mathscr{H}$ is regular and let $P$ be the projection of $\mathscr{H}^{\prime}$ onto $V \mathscr{H}$. If $V h=0$ for some $h \in \mathscr{H}$, then $[h, k]=[V h, V k]=0$ for all $k \in \mathscr{H}$, and so $V$ is injective. Thus we can define $U=V^{-1} P$, an operator from $\mathscr{H}^{\prime}$ to $\mathscr{K}$.

For all $h \in \mathscr{H}^{\circ}$ and $k \in \mathscr{H}^{\prime}$ we have

$$
[h, U k]=[V h, V U k]=[V h, P k]=[V h, k] .
$$

Consequently, by Lemma 3.2, $V$ is weakly continuous.

6. Wandering subspaces. Let $V$ be an isometry on $\mathscr{H}$. Following Halmos [5], we call a closed subspace $\mathscr{L}$ of $\mathscr{H}$ wandering for $V$ if it is nondegenerate and $V^{p} \mathscr{L} \perp V^{q} \mathscr{L}$ for every pair of integers $p, q \geqq 0, p \neq q$. Since $V$ is an isometry, $\mathscr{L}$ is wandering for $V$ if and only if it is nondegenerate and

$$
V^{n} \mathscr{L} \perp \mathscr{L} \quad \text { for } n=1,2, \cdots \text {. }
$$

Let $M_{+}(\mathscr{L})=\mathrm{V}_{n=0}^{\infty} V^{n} \mathscr{L}$ (i.e., the closed linear span of the subspaces $\left.V^{n} \mathscr{L}, n=0,1,2, \cdots\right)$.

Proposition 6.1. (i) $V M_{+}(\mathscr{L})=M_{+}(V \mathscr{L})$ and (ii) $\mathscr{L} \perp V M_{+}(\mathscr{L})$.

Proof. (i) follows from the definition of $M_{+}(\mathscr{L})$ and the fact that $V M_{+}(\mathscr{L})$ is closed (Lemma 5.1).

To prove (ii), note that $\mathscr{L} \perp V^{n+1} \mathscr{L}$ for each $n \geqq 0$, and so (by Corollary 2.4) $\mathscr{L} \perp M_{+}(V \mathscr{L})=V M_{+}(\mathscr{L})$.

THEOREM 6.2. If $\mathscr{L}$ is regular, then

$$
M_{+}(\mathscr{L})=\mathscr{L} \oplus V M_{+}(\mathscr{L})
$$




$$
V M_{+}(\mathscr{L})=M_{+}(\mathscr{L}) \ominus \mathscr{L} \text {. }
$$

Proof. Proposition 6.1 and the nondegeneracy of $\mathscr{L}$ show that the use of $\oplus$ in (6.1) is justified. Clearly, $M_{+}(\mathscr{L})$ is spanned by $\mathscr{L}$ and $V M_{+}(\mathscr{L})$. (6.1) follows from Theorem 4.6, since $\mathscr{L}$ is regular and $V M_{+}(\mathscr{L})$ is closed (Lemma 5.1).

(6.2) is a direct consequence of Lemma 2.1 , since $\mathscr{L}^{0}=\{0\}$.

CoROLlaRY 6.3. If $\mathscr{L}$ is regular, then for $n=1,2, \cdots$

$$
M_{+}(\mathscr{L})=\mathscr{L} \oplus V \mathscr{L} \oplus V^{2} \mathscr{L} \oplus \cdots \oplus V^{n-1} \mathscr{L} \oplus V^{n} M_{+}(\mathscr{L}) .
$$

When $\mathscr{H}$ is a Hilbert space, $\mathscr{L}$ is uniquely determined by $V$ and $M_{+}(\mathscr{L})$ :

$$
\mathscr{L}=M_{+}(\mathscr{L}) \ominus V M_{+}(\mathscr{L}) \text {. }
$$

The following example shows that this is not necessarily true when the inner product on $\mathscr{H}$ is not positive definite.

EXAMPLE 6.4. Let $\mathscr{H}$ be the space of sequences $x=\left\{x_{k}\right\}_{k \geqq 0}$ of complex numbers such that

$$
\|x\|^{2}=\sum_{k=0}^{\infty}\left|x_{k}\right|^{2}<\infty .
$$

For' two sequences $x$ and $y$ we define

$$
[x, y]=x_{0} \bar{y}_{0}-\sum_{k=1}^{\infty} x_{k} \bar{y}_{k} .
$$

The topology on $\mathscr{H}$ is that defined by the norm $\|\cdot\|$, and this topology is readily seen to be admissible.

Let $\mathscr{L}$ be the one-dimensional subspace of $\mathscr{H}$ spanned by the vector $l$, where $l_{0}=\sqrt{3}, l_{1}=2$ and $l_{k}=0$ for $k \geqq 2$. It is easily checked that $\mathscr{L}$ is regular.

For $x \in \mathscr{H}$ let $V x$ be the sequence $y \in \mathscr{H}$ with $y_{0}=2 x_{0}, y_{1}=$ $\sqrt{3} x_{0}$ and $y_{k}=x_{k-1}$ for $k>1$. Then $V$ is an isometry and $\mathscr{L}$ is wandering for $V$.

By writing down the condition that $z \perp V^{n} l$ for $n=0,1,2, \cdots$, it can be readily seen that $M_{+}(\mathscr{L})^{\perp}$ is spanned by the single vector $z$ with $z_{0}=1 / \sqrt{3}$ and $z_{k}=2^{-k}$ for $k \geqq 1$.

Let $\mathscr{L}^{\prime}$ be the one-dimensional subspace of $\mathscr{H}$ spanned by the vector $l^{\prime}=l+z$. Then $\mathscr{L}^{\prime}$ is regular and wandering for $V$. As before, it can be shown that $M_{+}\left(\mathscr{L}^{\prime}\right)^{\perp}$ is spanned by the same vector $z$ that spans $M_{+}(\mathscr{L})^{\perp}$. Consequently, $M_{+}\left(\mathscr{L}^{\prime}\right)=M_{+}(\mathscr{L})$, but $\mathscr{L}^{\prime} \neq \mathscr{L}$.

Note that in the preceding example $M_{+}(\mathscr{L})$ is a degenerate sub- 
space. In fact, since $M_{+}(\mathscr{L})^{\perp}$ is spanned by the vector $z$ and since

$$
[z, z]=1 / 3-\sum_{k=1}^{\infty} 4^{-k}=0,
$$

then $M_{+}(\mathscr{L})^{\perp} \subseteq M_{+}(\mathscr{L})$. The following theorem shows that the nondegeneracy of $M_{+}(\mathscr{L})$ is a necessary and sufficient condition for (6.3) to be true, provided $\mathscr{L}$ is regular.

THEOREM 6.5. If $\mathscr{L}$ is regular, then $\mathscr{L}=M_{+}(\mathscr{L}) \ominus V M_{+}(\mathscr{L})$ if and only if $M_{+}(\mathscr{L})$ is nondegenerate.

Proof. If $\mathscr{L}$ is regular, then Theorem 6.2 implies $M_{+}(\mathscr{L})=$ $\mathscr{L} \oplus V M_{+}(\mathscr{L})$. Thus, by Lemma 2.1,

$$
M_{+}(\mathscr{L}) \ominus V M_{+}(\mathscr{L})=\left[V M_{+}(\mathscr{L})\right]^{0} \oplus \mathscr{L} \text {. }
$$

Hence, $M_{+}(\mathscr{L}) \ominus V M_{+}(\mathscr{L})=\mathscr{L}$ if and only if $V M_{+}(\mathscr{L})$ is nondegenerate. Since $V$ is an isometry, this is equivalent to $M_{+}(\mathscr{L})$ being nondegenerate.

THEOREM 6.6. If $M_{+}(\mathscr{L})$ is regular, then $\mathscr{L}$ is regular. If $P$ is the projection of $\mathscr{L}$ onto $M_{+}(\mathscr{L})$, then $P-V P V^{*}$ is the projection of $\mathscr{H}$ onto $\mathscr{L}$.

Proof. If $M_{+}(\mathscr{L})$ is regular, then so is $V M_{+}(\mathscr{L})$, with the projection of $\mathscr{H}$ onto $V M_{+}(\mathscr{L})$ being $V P V^{*}$ (Theorem 5.2). As in the proof of Theorem 6.2 , we deduce that $M_{+}(\mathscr{L})=\mathscr{L} \oplus V M_{+}(\mathscr{L})$. Hence, as in the above proof, $\mathscr{L}=M_{+}(\mathscr{L}) \ominus V M_{+}(\mathscr{L})$, and the proof is completed by Corollary 4.5 .

THEOREM 6.7. If $\mathscr{L}$ is regular, then the isotropic part of $M_{+}(\mathscr{L})$ is

$$
M_{+}(\mathscr{L})^{0}=\bigcap_{n=0}^{\infty} V^{n} M_{+}(\mathscr{L})
$$

Proof. Suppose $h \in \bigcap_{n=0}^{\infty} V^{n} M_{+}(\mathscr{L})$. By Proposition 6.1 (ii) we have, for each $n \geqq 0, h \in V^{n+1} M_{+}(\mathscr{L}) \perp V^{n} \mathscr{L}$. Therefore $h \perp M_{+}(\mathscr{L})$. Since $h \in M_{+}(\mathscr{L})$, we conclude that

$$
\bigcap_{n=0}^{\infty} V^{n} M_{+}(\mathscr{L}) \subseteq M_{+}(\mathscr{L})^{0} .
$$

Conversely, assume $h \in M_{+}(\mathscr{L})^{0}$, and let us make the induction assumption that $h \in V^{n} M_{+}(\mathscr{L})$ (which is clearly true for $n=0$ ). Since $\mathscr{L}$ is regular, Theorem 6.2 implies 


$$
V^{n} M_{+}(\mathscr{L})=V^{n}\left(\mathscr{L} \oplus V M_{+}(\mathscr{L})\right)=V^{n} \mathscr{L} \oplus V^{n+1} M_{+}(\mathscr{L}) .
$$

We have assumed $h$ satisfies $h \perp M_{+}(\mathscr{L}) \supseteq V^{n} \mathscr{L}$, and $V^{n} \mathscr{L}$ is nondegenerate (because $\mathscr{L}$ is). Thus, by Lemma 2.1, $h \in V^{n+1} M_{+}(\mathscr{L})$, and hence, by induction, $h \in \bigcap_{n=0}^{\infty} V^{n} M_{+}(\mathscr{L})$.

Consequently, $M_{+}(\mathscr{L})^{0}=\bigcap_{n=0}^{\infty} V^{n} M_{+}(\mathscr{L})$.

7. Fourier coefficients in $M_{+}(\mathscr{L})$. We now assume that $\mathscr{L}$ is regular, with the projection of $\mathscr{H}$ onto $\mathscr{L}$ denoted by $P$.

Corollary 6.3 implies that for every $h \in M_{+}(\mathscr{L})$ there is a unique sequence $\left\{l_{n}\right\}_{n \geqq 0}$ of vectors in $\mathscr{L}$ such that, for each $n \geqq 1$,

$$
h-\sum_{k=0}^{n-1} V^{k} l_{k} \in V^{n} M_{+}(\mathscr{L}) .
$$

The vectors $l_{n}(n \geqq 0)$ satisfying (7.1) are known as the Fourier coefficients of $h$ in $M_{+}(\mathscr{L})$.

The Fourier coefficients can be written explicitly in terms of $h$ :

THEOREM 7.1. If $h \in M_{+}(\mathscr{L})$ then the Fourier coefficients of $h$ are given by

$$
l_{n}=P V^{* n} h, \quad n=0,1,2, \cdots .
$$

Proof. By Theorem 6.2,

$$
(I-P) M_{+}(\mathscr{L}) \subseteq M_{+}(\mathscr{L}) \Theta \mathscr{L}=V M_{+}(\mathscr{L}) \text {. }
$$

Hence if $l_{0}=P h,(7.1)$ is satisfied for $n=1$.

Suppose $h-\sum_{k=0}^{n=1} V^{k} l_{k}=V^{n} h^{\prime}$ for some $n \geqq 1, h^{\prime} \in M_{+}(\mathscr{L})$, and $l_{0}, l_{1}, \cdots, l_{n-1} \in \mathscr{L}$. Since $V$ is an isometry,

$$
V^{* n} h=\sum_{k=0}^{n-1} V^{* n-k} l_{k}+h^{\prime} .
$$

$\mathscr{L}$ is wandering for $V$ and so $V^{* m} \mathscr{L} \perp \mathscr{L}$ for all $m \geqq 1$. Thus $P V^{* n} h=P h^{\prime}$ and, letting $l_{n}=P V^{* n} h$, we have

$$
h-\sum_{k=0}^{n} V^{k} l_{k}=V^{n}(I-P) h^{\prime} \in V^{n+1} M_{+}(\mathscr{L}) .
$$

When the inner product on $\mathscr{H}$ is positive definite, $h$ can be expressed as the orthogonal sum

$$
h=\sum_{k=0}^{\infty} V^{k} l_{k} .
$$

In general, however, the Fourier coefficients do not determine $h$ uniquely. For example, the vector $z$ in Example 6.4 is in $M_{+}(\mathscr{C})^{0}$ 
and hence in $V^{n} M_{+}(\mathscr{L})$ for each $n \geqq 0$ (Theorem 6.7). Consequently, for $l_{n}=0$ for each $n$, (7.1) is satisfied both when $h=0$ and when $h=z$.

THEOREM 7.2. The map which associates to each $h \in M_{+}(\mathscr{L})$ its Fourier coefficients is injective if and only if $M_{+}(\mathscr{L})$ is nondegenerate.

Proof. From (7.1), $l_{n}=0$ for each $n$ if and only if

$$
h \in \bigcap_{n=0}^{\infty} V^{n} M_{+}(\mathscr{L})=M_{+}(\mathscr{L})^{0} \quad \text { (Theorem 6.7) }
$$

Since the map taking $h$ to $\left\{l_{n}\right\}_{n \geqq 0}$ is obviously linear, it is injective if and only if $M_{+}(\mathscr{L})$ is nondegenerate.

When $M_{+}(\mathscr{L})$ is nondegenerate, we still cannot expect to recover $h$ from its Fourier coefficients by means of (7.2). Indeed, even when $M_{+}(\mathscr{L})=\mathscr{K},(7.2)$ may not be valid, as the following example shows.

EXAMPLE 7.3. Let $\mathscr{H}$ be the space in Example 6.4, except that the inner product is given by

$$
[x, y]=x_{0} \bar{y}_{0}+\sum_{k=0}^{\infty} x_{2 k+1} \bar{y}_{2 k+1}-\sum_{k=1}^{\infty} x_{2 k} \bar{y}_{2 k} .
$$

Let $\mathscr{L}$ be the two-dimensional subspace of $\mathscr{C}$ spanned by the vectors $a$ and $b$, where $a_{0}=a_{2}=1, a_{k}=0$ for $k \neq 0,2$, and $b_{1}=b_{2}=$ $-1, b_{k}=0$ for $k \neq 1,2$. A vector $x$ is in $\mathscr{L}^{\perp}$ if and only if $x_{0}=$ $x_{1}=x_{2}$, and hence it is easily deduced that $\mathscr{L}$ is regular.

For $x \in \mathscr{C}$, let $V x$ be the vector $y \in \mathscr{H}$ with $y_{0}=y_{1}=x_{0}, y_{k}=$ $x_{k-2}$ for $k \geqq 2$. Then $V$ is an isometry and $\mathscr{L}$ is wandering for $V$. We have

$$
\left(V^{n} a\right)_{k}= \begin{cases}1 & \text { for } k=0,1, \cdots, 2 n ; k=2 n+2 \\ 0 & \text { for } k=2 n+1 ; k \geqq 2 n+3\end{cases}
$$

and

$$
\left(V^{n} b\right)_{k}=\left\{\begin{aligned}
0 & \text { for } k=0,1, \cdots, 2 n ; k \geqq 2 n+3 \\
-1 & \text { for } k=2 n+1,2 n+2 .
\end{aligned}\right.
$$

Hence it follows that $x \perp V^{m} \mathscr{L}$ for $m=0,1,2, \cdots, n-1$ if and only if $x_{0}=x_{1}=\cdots=x_{2 n-1}=x_{2 n}$. Consequently, only the zero vector is orthogonal to $M_{+}(\mathscr{L})$, and thus $M_{+}(\mathscr{L})=\mathscr{H}$.

Let $h$ be the vector with $h_{0}=1, h_{k}=0$ for $k \geqq 1$. Since 


$$
\left(\sum_{m=0}^{n-1} V^{m} b\right)_{k}=\left\{\begin{aligned}
-1 & \text { for } k=1,2, \cdots, 2 n-1,2 n \\
0 & \text { for } k=0 ; k>2 n
\end{aligned}\right.
$$

then

$$
\begin{aligned}
\left(h-\sum_{m=0}^{n-1} V^{m} b\right)_{k} & = \begin{cases}1 & \text { for } k=0,1, \cdots, 2 n-1,2 n \\
0 & \text { for } k>2 n\end{cases} \\
& =\left(V^{n} h\right)_{k} .
\end{aligned}
$$

Hence $h-\sum_{m=0}^{n-1} V^{m} b=V^{n} h \in V^{n} M_{+}(\mathscr{L})$, and we conclude that the Fourier coefficients of $h$ in $M_{+}(\mathscr{L})$ are all equal to $b$. However, $h \neq$ $\sum_{k=0}^{\infty} V^{k} b$, as the sum does not converge. Also note that although $h_{0}=1$, each of the partial sums $\sum_{k=0}^{n-1} V^{k} b$ has zeroth term equal to zero. If the inner product on $\mathscr{H}$ is positive definite, then

$$
[h, h]=\sum_{k=0}^{\infty}\left[l_{k}, l_{k}\right],
$$

but the above example, where $[h, h]=1$ and $\sum_{k=0}^{\infty}\left[l_{k}, l_{k}\right]=0$, shows this is not true in general.

8. Unilateral shifts. Wold decomposition. An isometry $V$ on $\mathscr{H}$ is called a unilateral shift if there exists in $\mathscr{H}$ a subspace $\mathscr{L}$ which is wandering for $V$ such that $M_{+}(\mathscr{L})=\mathscr{H}$. This subspace $\mathscr{L}$, called generating for $V$, is necessarily regular (Theorem 6.6) and is uniquely determined by $V$ :

$$
\mathscr{L}=(V \mathscr{H})^{\perp} \quad(\text { Theorem 6.5) }
$$

(cf. [5]).

In Hilbert space, a unilateral shift is determined up to unitary equivalence by the dimension of $\mathscr{L}$. In general, however, it is possible to have two unilateral shifts acting on isomorphic spaces that are not unitarily equivalent, even though their generating subspaces are isomorphic.

EXAMPLE 8.1. Let $\mathscr{H}$ be the space in Example 6.4, except that the inner product is defined by

$$
[x, y]=\sum_{k=0}^{\infty} x_{2 k} \bar{y}_{2 k}-\sum_{k=0}^{\infty} x_{2 k+1} \bar{y}_{2 k+1} .
$$

Let $\mathscr{L}$ be the two-dimensional subspace of $\mathscr{H}$ spanned by the vectors $a$ and $b$, where $a_{0}=a_{1}=1, a_{k}=0$ for $k \neq 0,1$, and $b_{1}=b_{2}=1$, $b_{k}=0$ for $k \neq 1,2$.

For $x \in \mathscr{H}$, let $V x$ be the vector $y \in \mathscr{H}$ with $y_{0}=y_{1}=x_{0}$ and $y_{k}=x_{k-2}$ for $k \geqq 2$. Then $V$ is an isometry and $\mathscr{L}$ is wandering for $V$. 
It is readily checked that a vector $x \in \mathscr{H}$ is orthogonal to $V^{m} \mathscr{L}$ for $m=0,1,2, \cdots, n-1$ if and only if $x_{0}=x_{1}=\cdots=x_{2 n}$, and therefore, as in Example 7.3, $M_{+}(\mathscr{L})=\mathscr{H}$. Thus $V$ is a unilateral shift on $\mathscr{H}$ with generating subspace $\mathscr{L}$.

Another unilateral shift on $\mathscr{H}$ is the isometry $V^{\prime}$ defined by $V^{\prime} x=y$, where $y_{0}=y_{1}=0$ and $y_{k}=x_{k-2}$ for $k \geqq 2$. The operator $\phi$ defined on $\mathscr{L}$ by $\phi a=c$, where $c_{0}=c_{1}=1 / \sqrt{2}$ and $c_{k}=0$ for $k \geqq 2$, and $\phi b=d$, where $d_{0}=-1 / \sqrt{2}, d_{1}=1 / \sqrt{2}$ and $d_{k}=0$ for $k \geqq 2$, is a unitary operator from $\mathscr{L}$ onto the generating subspace for $V^{\prime}$. But for any $x \in \mathscr{K}$ with $x_{0} \neq 0$, we have (for $n=0,1,2, \cdots$ )

$$
\left\|V^{\prime n} x\right\|=\|x\|,
$$

whereas $\left\|V^{n} x\right\| \rightarrow \infty$ as $n \rightarrow \infty$. Thus, despite having isomorphic generating subspaces, $V$ and $V^{\prime}$ are not unitarily equivalent.

In Hilbert space, the property $V^{* n} x \rightarrow 0$ for all $x$ characterizes those isometries that are unilateral shifts [5]. This does not apply in general, as the vector $h$ in Example 7.3 has $V^{* n} h=h$ for $n=$ $0,1, \cdots$.

We can prove a Wold decomposition theorem for isometries, but it does not have the generality of the corresponding result in Hilbert space (cf. [11, Theorem I.1.1]).

THeOREM 8.2. Let $V$ be an isometry on $\mathscr{H}$ and let $\mathscr{L}=(V \mathscr{H})$. In order to have the orthogonal sum $\mathscr{H}=\mathscr{C}_{0} \oplus \mathscr{H}_{1}$, where $\mathscr{H}_{0}$ and $\mathscr{H}_{1}$ are invariant for $V, V \mid \mathscr{H}_{0}$ is a unilateral shift and $V \mid \mathscr{H}_{1}$ is unitary, it is necessary and sufficient that $M_{+}(\mathscr{L})$ be regular. In that case,

$$
\mathscr{K}_{0}=M_{+}(\mathscr{L})
$$

and

$$
\mathscr{H}_{1}=\bigcap_{n=0}^{\infty} V^{n} \mathscr{K}
$$

Proof. Suppose that $M_{+}(\mathscr{L})$ is regular, and let $\mathscr{C}_{0}=M_{+}(\mathscr{L})$, $\mathscr{K}_{1}=M_{+}(\mathscr{L})^{\perp}$. Then we have $\mathscr{H}=\mathscr{C}_{0} \oplus \mathscr{C}_{1}$. It is clear that $\mathscr{C}_{0}$ is invariant for $V$ and $V \mid \mathscr{C}_{0}$ is a unilateral shift. By a similar argument to that in [5, Lemma 1], $\mathscr{C}_{1}=\bigcap_{n=0}^{\infty} V^{n} \mathscr{C}$, and so $V \mathscr{C}_{1}=$ $\mathscr{H}_{1}$. Thus, $V \mid \mathscr{H}_{1}$ is unitary.

Conversely, suppose $\mathscr{H}_{0}$ and $\mathscr{H}_{1}$ are invariant for $V$, where $\mathscr{Y}=$ $\mathscr{K}_{0} \oplus \mathscr{H}_{1}, V \mid \mathscr{K}_{0}$ is a unilateral shift, and $V \mid \mathscr{H}_{1}$ is unitary. Since $V \mathscr{H}_{1}=\mathscr{H}_{1}$, we have $\mathscr{L}=(V \mathscr{H})^{\perp}=\mathscr{H} \cap \ominus V \mathscr{H}_{0}$, and thus $\mathscr{L}$ is the generating subspace for the unilateral shift $V \mid \mathscr{C}_{0}$. Consequently, $M_{+}(\mathscr{L})=\mathscr{K}_{0}$, and since $\mathscr{H}=\mathscr{H}_{0} \oplus \mathscr{H}_{1}, M_{+}(\mathscr{L})$ is regular. 
9. The space $M(\mathscr{L})$. Fourier coefficients in $M(\mathscr{L})$. Let $V$ be a unitary operator on $\mathscr{H}$ and let $\mathscr{L}$ be a wandering subspace for $V$. Then $\mathscr{L}$ is nondegenerate and $V^{p} \mathscr{L} \perp V^{q} \mathscr{L}$ for all integers $p$ and $q$. We define

$$
M(\mathscr{L})=\bigvee_{n=-\infty}^{\infty} V^{n} \mathscr{L} .
$$

It is clear that $M(\mathscr{L})$ reduces $V$ and that $M(\mathscr{L})=M(V \mathscr{L})$.

The following can be proved in the same manner as Theorem 6.2.

THEOREM 9.1. If $\mathscr{L}$ is regular and $n$ is an integer, then

$$
M(\mathscr{L})=V^{n} \mathscr{L} \oplus \bigvee\left\{V^{k} \mathscr{L}: k \neq n\right\} .
$$

COROLLARY 9.2. If $\mathscr{L}$ is regular and $m$ and $n$ are integers such that $m \leqq n$, then $M(\mathscr{L})=V^{m} \mathscr{L} \oplus V^{m+1} \mathscr{L} \oplus \cdots \oplus V^{n} \mathscr{L} \oplus \bigvee\left\{V^{k} \mathscr{L}: k>n, k<m\right\}$.

Consequently, if $\mathscr{L}$ is regular and $h \in M(\mathscr{L})$, there is a unique sequence $\left\{l_{n}\right\}_{n=-\infty}^{\infty}$ of vectors in $\mathscr{L}$ such that for all integers $m$ and $n$, with $m \leqq n$,

$$
h-\sum_{k=m}^{n} V^{k} l_{k} \in \mathrm{V}\left\{V^{j} \mathscr{L}: j>n, j<m\right\} .
$$

The vectors $l_{n}$ satisfying (9.1) are known as the Fourier coefficients of $h$ in $M(\mathscr{L})$. Clearly, if $h \in M_{+}(\mathscr{L})$, then $l_{n}=0$ for $n<0$ and $\left\{l_{n}\right\}_{n \geqq 0}$ is the sequence of Fourier coefficients of $h$ in $M_{+}(\mathscr{L})$.

We now assume that $\mathscr{L}$ is regular with the projection of $\mathscr{C}$ onto $\mathscr{L}$ denoted by $P$. The following analogue of Theorem 7.1 is given here without proof (see [10, Theorem II.7.4]).

TheOREm 9.3. If $h \in M(\mathscr{L})$ then the Fourier coefficients of $h$ are given by

$$
l_{n}=P V^{* n} h, \quad n=0, \pm 1, \pm 2, \cdots .
$$

10. The space $M_{-}(\mathscr{P})$. Let $\mathscr{L}$ be a wandering subspace for the unitary operator $V$ on $\mathscr{C}$. We define

$$
M_{-}(\mathscr{L})=\overrightarrow{\mathrm{V}}_{n=-\infty} V^{n} \mathscr{L} .
$$

From the above definition, Theorem 4.6, and Lemma 2.1 we obtain the following decomposition of $M(\mathscr{L})$.

Theorem 10.1. If $M_{+}(\mathscr{L})$ is regular, then 


$$
M(\mathscr{L})=M_{-}(\mathscr{L}) \oplus M_{+}(\mathscr{L})
$$

and

$$
M_{-}(\mathscr{L})=M(\mathscr{L}) \ominus M_{+}(\mathscr{L})
$$

Note that $M_{-}(\mathscr{L})$ is the same as the space $M_{+}\left(V^{-1} \mathscr{L}\right)$, formed with respect to the unitary $V^{-1}$. We can therefore obtain directly from the properties proved for $M_{+}(\mathscr{L})$ analogous properties for $M_{-}(\mathscr{L})$. We start with a corollary to Theorem 10.1.

Corollary 10.2. If $M_{-}(\mathscr{L})$ is regular, then

$$
M(\mathscr{L})=M_{-}(\mathscr{L}) \oplus M_{+}(\mathscr{L})
$$

and

$$
M_{+}(\mathscr{L})=M(\mathscr{L}) \ominus M_{-}(\mathscr{L})
$$

THeOREM 10.3. If $M_{-}(\mathscr{L})$ is regular, then $\mathscr{L}$ is regular. If $P$ is the projection of $\mathscr{H}$ onto $M_{-}(\mathscr{L})$, then $V P V^{*}-P$ is the projection of $\mathscr{H}$ onto $\mathscr{L}$.

Proof. Apply Theorem 6.6.

THEOREM 10.4. If $\mathscr{L}$ is regular, then

$$
V M_{-}(\mathscr{L})=M_{-}(\mathscr{L}) \oplus \mathscr{L}
$$

and

$$
M_{-}(\mathscr{L})=V M_{-}(\mathscr{L}) \ominus \mathscr{L}
$$

Proof. Apply Theorem 6.2.

CoROllary 10.5. If $\mathscr{L}$ is a regular, then for $n=1,2, \cdots$

$$
M_{-}(\mathscr{L})=V^{-1} \mathscr{L} \oplus V^{-2} \mathscr{L} \oplus \cdots \oplus V^{-n} \mathscr{L} \oplus V^{-n} M_{-}(\mathscr{L}) .
$$

Hence, if $\mathscr{L}$ is regular and $h \in M_{-}(\mathscr{L})$, there is a unique sequence $\left\{l_{n}\right\}_{n<0}$ of vectors in $\mathscr{L}$ such that, for all $n<0$,

$$
h-\sum_{k=n}^{-1} V^{k} l_{k} \in V^{n} M_{-}(\mathscr{L}) \text {. }
$$

The vectors $l_{n}(n<0)$ satisfying (10.1) are known as the Fourier coefficients of $h$ in $M_{-}(\mathscr{L})$, and these obviously coincide with the Fourier coefficients (with negative index) of $h$ in $M(\mathscr{L})$.

Let $P$ be the projection of $\mathscr{H}$ onto $\mathscr{L}$. For completeness, we 
state the following theorem (cf. Theorem 7.1 and Theorem 9.3).

THEOREM 10.6. If $h \in M_{-}(\mathscr{L})$ then the Fourier coefficients of $h$ are given by

$$
l_{n}=P V^{* n} h, \quad n=-1,-2, \cdots .
$$

When $M_{+}(\mathscr{L})$ or $M_{-}(\mathscr{L})$ is regular we get a strengthening of Corollary 9.2 which more closely resembles Corollary 6.3.

THEOREM 10.7. If $M_{+}(\mathscr{L})$ or $M_{-}(\mathscr{L})$ is regular, then for each $m, n \geqq 0$, we have

$$
\begin{aligned}
M(\mathscr{L})= & V^{-m} M_{-}(\mathscr{L}) \oplus V^{-m} \mathscr{L} \\
& \oplus V^{-m+1} \mathscr{L} \oplus \cdots \oplus V^{n-1} \mathscr{L} \oplus V^{n} M_{+}(\mathscr{L}) .
\end{aligned}
$$

Proof. If $M_{+}(\mathscr{L})$ is regular, then by Theorem $6.6 \mathscr{L}$ is regular. Hence, by Corollary 6.3 and Corollary 10.5, we have

$$
M_{+}(\mathscr{L})=\mathscr{L} \oplus V \mathscr{L} \oplus \cdots \oplus V^{n-1} \mathscr{L} \oplus V^{n} M_{+}(\mathscr{L})
$$

and

$$
M_{-}(\mathscr{L})=V^{-1} \mathscr{L} \oplus V^{-2} \mathscr{L} \oplus \cdots \oplus V^{-m} \mathscr{L} \oplus V^{-m} M_{-}(\mathscr{L}) .
$$

The same equations are obtained when $M_{-}(\mathscr{L})$ is regular (by Theorem 10.3), and the proof is completed by using Theorem 10.1 or Corollary 10.2 .

When $M_{+}(\mathscr{L})$ or $M_{-}(\mathscr{L})$ is regular, we can rewrite (9.1) as

$$
h-\sum_{k=-m}^{n-1} V^{k} l_{k} \in V^{-m} M_{-}(\mathscr{L}) \oplus V^{n} M_{+}(\mathscr{L}),
$$

where $m, n \geqq 0$.

11. Fourier representations. Let $V$ be an isometry on $\mathscr{K}$, and suppose $\mathscr{L}$ is a wandering subspace for $V$, with $M_{+}(\mathscr{L})$ regular. Then $\mathscr{L}$ is regular (Theorem 6.6), and every vector $h \in M_{+}(\mathscr{L})$ has a sequence $\left\{l_{n}\right\}_{n \geqq 0}$ of Fourier coefficients given by Theorem 7.1. The Fourier representation of $M_{+}(\mathscr{L})$ is the map $\Phi$ which associates to every $h \in M_{+}(\mathscr{L})$ the function $\Phi h$, where

$$
(\Phi h)(\lambda)=\sum_{n=0}^{\infty} \lambda^{n} l_{n}
$$

Th is defined for all complex numbers $\lambda$ for which the series (11.1) converges, and takes values in the space $\mathscr{L}$. The action of $V$ on $M_{+}(\mathscr{L})$ corresponds (via the Fourier representation) to multiplication by the independent variable $\lambda$ on $\Phi M_{+}(\mathscr{L})$. 
If the topology on $\mathscr{L}$ can be defined by a norm $\|\cdot\|$, and if $\mathscr{L}$ is separable, then we define $H^{2}(\mathscr{L})$ as the space of all functions $u$, defined and analytic on the open unit disk, with values in $\mathscr{L}$, such that

$$
\|u\|^{2}=\lim _{r \rightarrow 1-} 1 / 2 \pi \int_{0}^{2 \pi}\left\|u\left(r e^{i t}\right)\right\|^{2} d t
$$

exists. The inner product on $H^{2}(\mathscr{L})$ is given by

$$
[u, v]=\lim _{r \rightarrow 1-} 1 / 2 \pi \int_{0}^{2 \pi}\left[u\left(r e^{i t}\right), v\left(r e^{i t}\right)\right] d t .
$$

In Hilbert space, the shift $V \mid M_{+}(\mathscr{L})$ depends (up to unitary equivalence) only on the generating subspace $\mathscr{L}$, and $\Phi$ is a unitary map from $M_{+}(\mathscr{L})$ onto $H^{2}(\mathscr{L})$ (cf. [5], [11, Sec. V.3]). The situation is different here, as Example 8.1 shows that the generating subspace does not uniquely determine the shift, and so $\Phi M_{+}(\mathscr{L})$ can be different from $H^{2}(\mathscr{L})$. Indeed, the vector $h$ in Example 7.3 has

$$
(\Phi h)(\lambda)=\sum_{n=0}^{\infty} \lambda^{n} b=(1-\lambda)^{-1} b,
$$

and clearly $\Phi h \notin H^{2}(\mathscr{L})$.

If $V$ is unitary, we can also discuss the Fourier representation $\Phi$ of $M(\mathscr{L})$. If $h \in M(\mathscr{L})$ and $\left\{l_{n}\right\}_{n=-\infty}^{\infty}$ is the sequence of Fourier coefficients of $h$ in $M(\mathscr{L})$, then $\Phi h$ is the function pair $(u, v)$, where

$$
u(\lambda)=\sum_{n=-\infty}^{-1} \lambda^{n} l_{n}
$$

and $v(\lambda)$ is defined by the right hand side of (11.1) (cf. [10, Sec. IV.4]). The function $u$ is defined on some neighborhood of infinity, and $v$ is defined on some neighborhood of zero.

In Hilbert space, the Fourier representation is taken so that $\Phi$ is a unitary map from $M(\mathscr{L})$ onto $L^{2}(\mathscr{L})$, the space of all measurable functions defined on the unit circle, taking values in $\mathscr{L}$, and having square integrable norms (cf. [5], [11, Sec. V.3]). As with the Fourier representation of $M_{+}(\mathscr{L})$, this is not valid in general. However, if $\mathscr{L}$ is normed and separable, and if we have, for each $h \in M(\mathscr{L})$

$$
\sum_{n=-\infty}^{\infty}\left\|l_{n}\right\|^{2}<\infty, \text { and }[h, h]=\sum_{n=-\infty}^{\infty}\left[l_{n}, l_{n}\right]
$$

then we do obtain a representation of $M(\mathscr{L})$ as the space $L^{2}(\mathscr{L})$, with inner product

$$
[f, g]=1 / 2 \pi \int_{0}^{2 \pi}\left[f\left(e^{i t}\right), g\left(e^{i t}\right)\right] d t .
$$

This can be done by replacing $u$ and $v$ by their radial limits (a.e.) 


$$
u\left(e^{i t}\right)=\lim _{r \rightarrow 1+} u\left(r e^{i t}\right) \text { and } v\left(e^{i t}\right)=\lim _{r \rightarrow 1-} v\left(r e^{i t}\right)
$$

and identifying the pair $(u, v)$ with the $L^{2}$ function

$$
u\left(e^{i t}\right)+v\left(e^{i t}\right)=\sum_{n=-\infty}^{\infty} e^{i n t} l_{n} .
$$

Because the spaces $M_{+}(\mathscr{L})$ and $M(\mathscr{L})$ can not always be represented as $H^{2}$ or $L^{2}$ spaces, Fourier representations are not as powerful a tool in studying shifts as they are in Hilbert space. One approach is to restrict our attention to shifts for which it is known that $\Phi$ is a unitary operator from $M_{+}(\mathscr{L})$ to $H^{2}(\mathscr{L})$ (or from $M(\mathscr{L})$ to $L^{2}(\mathscr{L})$ ). This is done in [3] and [10, Chapter IV], where dilations of operators with bounded characteristic function are studied.

Another approach is to try to determine the types of function spaces that can occur if $\Phi$ is to be unitary. This is done in [10, Chapter VI], where dilations of some operators with unbounded characteristic function are studied. The situation is complicated by the fact that the inner product on the function space $\Phi M_{+}(\mathscr{L})$ need not be given by (11.2). This can be seen by considering the vector $h$ in Example 7.3, which has $[h, h]=1$, whereas (11.2) would assign the value zero to the inner product $[\Phi h, \Phi h]$ (see the remark following Example 7.3).

\section{REFERENCES}

1. J. Bognár, Indefinite inner product spaces, Springer-Verlag, New York (1974).

2. Ch. Davis, J-unitary dilation of a general operator, Acta Sci. Math., 31 (1970), 75-86.

3. Ch. Davis and C. Foias, Operators with bounded characteristic function and their J-unitary dilation, Acta Sci. Math., 32 (1971), 127-139.

4. N. Dunford and J. Schwartz, Linear operators, Part I, Interscience, New York (1958).

5. P. R. Halmos, Shifts on Hilbert spaces, J. reine angew. Math., 208 (1961), 102-112.

6. I. S. Iohvidov, Boundedness of J-isometric operators, Amer. Math. Soc. Transl.,

(2) 47 (1965), 67-71. (Translated from Uspehi Mat. Nauk, 16, no. 4 (1961), 167-170.)

7. - G-isometric and J-semiunitary operators in Hilbert space (In Russian), Uspehi Mat. Nauk, 20, no. 3 (1965), 175-181.

8. M. G. Krein, Introduction to the geometry of indefinite J-spaces and to the theory of operators in those spaces, Amer. Math. Soc. Transl., (2) 93 (1970), 103-176. (Translated from Second mathematical summer school, Part I, 15-92, Kiev: Naukova Dumka 1965.) 9. M. G. Krein and Ju. L. Šmul'jan, J-polar representations of plus-operators, Amer. Math. Soc. Transl., (2) 85 (1969), 115-143. (Translated from Mat. Issled., 1 (1966), no. 1, 172-210.)

10. B. W. McEnnis, Characteristic functions and the geometry of dilation spaces, $\mathrm{Ph}$.

D. thesis, University of Toronto (1977).

11. B. Sz.-Nagy and C. Foiaș, Harmonic Analysis of Operators on Hilbert space, North Holland, Amsterdam-London (1970).

Received April 10, 1978.

UNIVERSITY OF MISSOURI-ROLLA

ROLLA, MO 65401 


\section{PACIFIC JOURNAL OF MATHEMATICS}

EDITORS

DONALD BABBITT (Managing Editor)

University of California

Los Angeles, California 90024

HUGo RossI

University of Utah

Salt Lake City, UT 84112

C. C. MOORE

J. DUGUNDJI

Department of Mathematics

University of Southern California

Los Angeles, California 90007

R. FinN AND J. MiLgraM

Stanford University

Stanford, California 94305

University of California

Berkeley, CA 94720

\section{ASSOCIATE EDITORS}

E. F. BrCKENBACH

B. H. NeUmanN

F. WOLF

K. YoshidA

\section{SUPPORTING INSTITUTIONS}

UNIVERSITY OF BRITISH COLUMBIA

UNIVERSITY OF SOUTHERN CALIFORNIA

CALIFORNIA INSTITUTE OF TECHNOLOGY

STANFORD UNIVERSITY

UNIVERSITY OF CALIFORNIA

UNIVERSITY OF HAWAII

MONTANA STATE UNIVERSITY

UNIVERSITY OF TOKYO

UNIVERSITY OF NEVADA, RENO

UNIVERSITY OF UTAH

NEW MEXICO STATE UNIVERSITY

WASHINGTON STATE UNIVERSITY

OREGON STATE UNIVERSITY

UNIVERSITY OF WASHINGTON

UNIVERSITY OF OREGON 


\section{Pacific Journal of Mathematics}

\section{Vol. 81, No. $1 \quad$ November, 1979}

Thomas E. Armstrong, Simplicial subdivision of infinite-dimensional compact cubes ..................................... 1

Herbert Stanley Bear, Jr., Approximate identities and pointwise convergence ................................

Richard David Bourgin, Partial orderings for integral representations on convex sets with the Radon-Nikodým property..................

Alan Day, Herbert S. Gaskill and Werner Poguntke, Distributive lattices

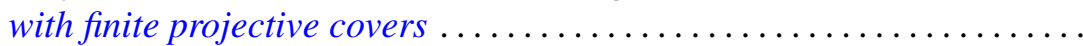

Heneri Amos Murima Dzinotyiweyi and Gerard L. G. Sleijpen, A note on measures on foundation semigroups with weakly compact orbits ......

Ronald James Evans, Resolution of sign ambiguities in Jacobi and Jacobsthal sums ...................................

John Albert Fridy, Tauberian theorems via block dominated matrices ......

Matthew Gould and Helen H. James, Automorphism groups retracting onto

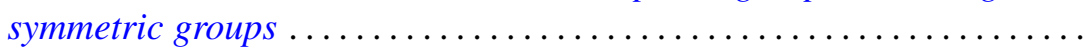

Kurt Kreith, Nonlinear differential equations with monotone solutions . . . . 101

Brian William McEnnis, Shifts on indefinite inner product spaces........ 113

Joseph B. Miles, On entire functions of infinite order with radially

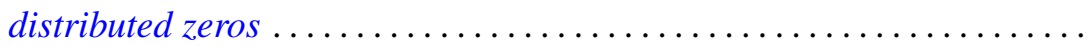

Janet E. Mills, The idempotents of a class of 0-simple inverse semigroups ...............................

Edward Jean Moulis, Jr., Generalizations of the Robertson functions ...

Richard A. Moynihan and Berthold Schweizer, Betweenness relations in probabilistic metric spaces.......................

Stanley Ocken, Perturbing embeddings in codimension two ....

Masilamani Sambandham, On the average number of real zeros of a class of random algebraic curves.

Jerry Searcy and B. Andreas Troesch, A cyclic inequality and a related eigenvalue problem.

Roger R. Smith and Joseph Dinneen Ward, $M$-ideals in $B\left(l_{p}\right)$...

Michel Talagrand, Deux généralisations d'un théorème de I. Namioka ..

Jürgen Voigt, $O n Y$-closed subspaces of $X$, for Banach spaces $X \subset Y$;

existence of alternating elements in subspaces of $C(J)$

Sidney Martin Webster, On mapping an $n$-ball into an $(n+1)$-ball in complex spaces

David J. Winter, Triangulable subalgebras of Lie p-algebras ... 